\title{
ON THE RECONSTRUCTION OF S-CHANNEL HELICITY AMPLITUDES FROM A COMPLETE SET OF MEASUREMENTS*
}

\author{
W. de BOER' \\ University of Michigan, Ann Arbor, Michigan 48104, U.S.A. \\ and
}

J. SOFFER+

Argonne National Laboratory, Argonne, Illinois 60439, U.S.A.

Received 1 April 1976

\begin{abstract}
The problem of reconstruction of the pp elastic scattering amplitudes from a complete set of measurements has been studied, taking into account the new experiments, which have become feasible with the advent of a high energy polarized beam at Argonne National Laboratory. Several experiments have been performed or proposed and we show analytically, that by performing two additional experiments ( $R$ and $A$ type measurements), one can obtain all the amplitudes without ambiguities. The uncertainties in the amplitudes, introduced by experimental errors, are also calculated.
\end{abstract}

\section{Introduction}

For particle reactions with a arbitrary spins it is a central problem to select a feasilbe set of experiments, such that it will be possible to extract the scattering amplitudes, which contain needless to say, the relevant physical information.

Extensive literature exists on how many experiments are needed ${ }^{1}$ ) and how to reconstruct the amplitudes from a complete set of observables, both for $N N^{2}$ ) and $\pi N^{3}$ ) scattering.

However, in case of $N N$ scattering the specific solutions are not obtained from very practical measurements. If one considers only practical measurements, then an analytic reconstruction becomes more difficult. At present sufficient measurements on pp elastic scattering have been done to determine the moduli of the five complex amplitudes in the transversity basis. This will be discussed in more detail later. These experiments do not form a complete set, since the phases between the amplitudes are still undetermined. To obtain these phases one can choose between many experiments and it is relevant to ask, which minimum set of experiments would give maximum constraints on the amplitudes.

We show analytically that in principle only four more observables are needed to determine the three natural parity exchange amplitudes without ambiguities, while the relative phases between the two unnatural parity exchange amplitudes can be obtained if the experimental errors are small enough. If one uses a polarized beam in combination with a polarized target, then these four observables require only two experimental set-ups.

The remaining unknown phase between the natural and unnatural parity exchange amplitudes requires two more measurements. One possible experiment, from which these two observables can be obtained, has been proposed recently ${ }^{4}$ ).

To obtain the amplitudes from measured observables, one has to solve a set of simultaneous, quadratic equations with complex unknowns. Our way of solving such a set of equations will be demonstrated first in the simple case of $\pi N$ elastic scattering, in which case there are only two amplitudes involved. We will then apply the method to a specific numerical example in the more complicated case of $N N$ scattering, taking into account the exprimental errors.

\section{General considerations}

Let us consider a particle reaction with arbitrary spins and described by $n$ complex amplitudes $a_{i}(i=1, \ldots, n)$. The complete knowledge of this reaction requires, up to one phase, $2 n-1$ real functions. However, one can

* Work performed under the auspices of the U.S. Energy Research and Development Administration.

$\dagger$ Present address: Max-Planck-Institut fur Physik and Astrophysik, Fohringer Ring 6, 8000 München-23, W.-Germany.

+ Permanent address: Centre de Physique Théorique CNRS, 31 Chemin Aiguier, Marseille Cedex 2, France. 
perform $n^{2}$ independent measurements $I_{i}\left(i=1, \ldots, n^{2}\right)$, which are linear combinations of bilinear products of amplitudes

$I_{k}=\sum_{i j} \alpha_{i j}^{k}\left\{\begin{array}{l}\mathrm{Re} \\ \operatorname{Im}\end{array}\right\} a_{i} a_{j}^{*}$,

where $\alpha_{i j}^{k}$ are known real numbers and $\left\{\begin{array}{l}\mathrm{Re} \\ \mathrm{Im}\end{array}\right\}$ means the real or imaginary part of the complex number $a_{i} a_{j}^{*}$. Conversely, for each $i$ and $j$ one has

$a_{i} a_{j}^{*}=\sum_{k} \beta_{k}^{i j} I_{k}$

where $\beta_{k}^{i j}$ are known complex coefficients. It is very useful to consider the Hermitian positive $n \times n$ matrix $M_{i j}=a_{i} a_{j}^{*}$, expressed in terms of the observables. All the $I_{i}$ are not independent, but they are related by $(n-1)^{2}$ quadratic relations ${ }^{5}$ ):

$\left|M_{i j}\right|^{2}=M_{i i} M_{j j}, \quad(i \neq j)$,

$M_{i j} M_{j k}=M_{j j} M_{i k}, \quad(i \neq j \neq k)$.

These equalities should be satisfied by experimental data and can provide some cross checks on various independent measurements. Obviously, we should also have

$\left\{\begin{array}{l}\mathrm{Re} \\ \mathrm{Im}\end{array}\right\} M_{i j} \leqslant \sqrt{ } M_{i i} \sqrt{ } M_{j j}$

which can give upper bounds on some observables. The usefulness of these relations will be illustrated later.

\section{3. $\pi \mathrm{N}$ elastic scattering}

This reaction involves only two helicity amplitudes, the non-flip $f_{++}$and the flip $f_{+_{-}}$, since pions are spinless. The four possible independent measurements are given in terms of the amplitudes in appendix 1. The laboratory observables $0(a ; b)$ are defined with respect to a frame for each particle, such that $l$ is along the direction of motion, $\boldsymbol{n}$ is normal to the scattering plane and $\boldsymbol{s}=\boldsymbol{n} \times \boldsymbol{l}$ (see fig. 1).

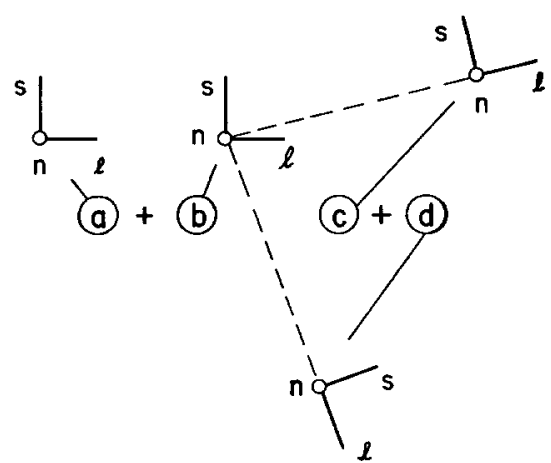

Fig. 1. Definition of the coordinate systems for the in- and outgoing particles in the reaction $a+b \rightarrow c+d$. The vector $n$, denoted by a circle, points out of the paper.

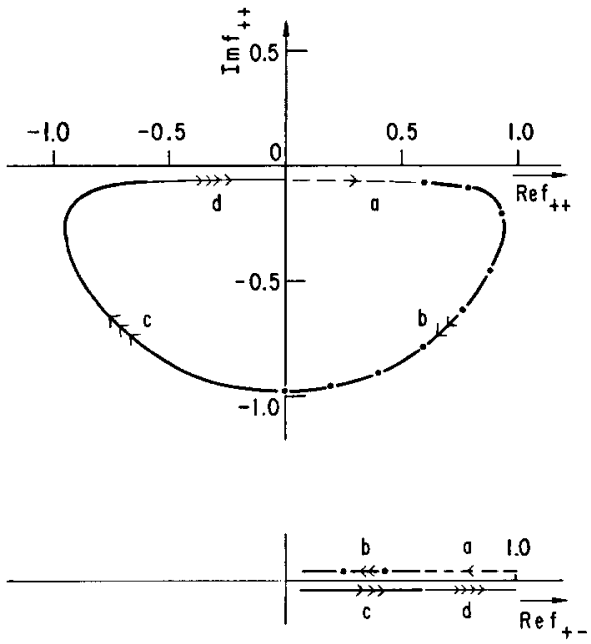

Fig. 2. Restrictions on the amplitudes $f_{++}$and $f_{+-}$in $\pi^{+} \mathrm{p}$ elastic scattering, imposed by the differential cross-section and the polarization. The phase of $f_{+-}$is assumed to be zero. When $f_{++}$has some possible value along the path $a b c d, f_{+}$is bound by eqs. (6) and (7) to have a corresponding value on the lower plot. 
Suppose we have only measured the differential cross section $\sigma$ and the polarization $P$ and we ask ourselves what do we know about $f_{++}$and $f_{+-}$. Let us take $f_{+-}$along the real axis and denote by $\phi$ the relative phase between $f_{++}$and $f_{+}$. Then we know that the sum and the product of the moduli squared is given by (see appendix 1):

$$
\begin{aligned}
& \left|f_{++}\right|^{2}+\left|f_{+-}\right|^{2}=\sigma \\
& \left|f_{++}\right|^{2}\left|f_{+-}\right|^{2}=\frac{P^{2}}{4 \sin ^{2} \phi} .
\end{aligned}
$$

Therefore the modulus squared of one of the amplitudes, denoted by $\rho$, must satisfy the equation

$$
\rho^{2}-\sigma \rho+\frac{P^{2}}{4 \sin ^{2} \phi}=0,
$$

since the solutions of this quadratic equation give immediately eqs. (6a) and (6b). Since $\rho$ has to be real, the discriminant of this equation has to be positive or

$\sin ^{2} \phi \geqslant(P / \sigma)^{2}$,

which gives a range for $\phi$. From eq. (7b) we find also $\sigma>P$ which is obviously satisfied. Although of no use in this simple case, this kind of inequality, which was written in a general form in eq. (5), will provide non-trivial bounds for more complicated reactions. Eq. (7a) is an interesting relation between $\rho$ and $\phi$, which gives restrictions on the possible amplitudes, as illustrated in fig. 2 , for the reaction $\pi^{+} \mathrm{p}$ at $p_{\mathrm{lab}}=6 \mathrm{GeV} / c$ and $-t=0.3(\mathrm{GeV} / c)^{2}$ for which $P=0.175 \pm 0.01^{6}$ ). First of all eq. (7b) implies $-173^{\circ} \leqslant \phi \leqslant-7^{\circ *}$. For each value of $\phi$ there are two possible values of $\rho$, one is $\left|f_{++}\right|^{2}$ and the other is $\left|f_{+-}\right|^{2}$ and we have explicitly marked corresponding paths for $f_{++}$and $f_{+-}$in fig. 2 . There is obviously a symmetry $\phi \rightarrow \pi-\phi$. Fig. 2 summarizes what we know about the amplitudes, when $\sigma$ and $P$ have been measured. In order to obtain more information about the phase, one needs one more measurement, for example the $R$ parameter, which has been measured to be $-0.2 \pm 0.11^{7}$ ). Needless to recall that the magnitude of the $A$ parameter is no longer independent, because of the well known relation

$\sigma^{2}=P^{2}+R^{2}+A^{2}$

which is a special case of eq. (3) (see also appendix 1). Although this relation gives the magnitude of $A$, we do not know its sign, but we expect the non-flip amplitude to be dominant over the flip amplitude, which implies $A>0$. Indeed, $A$ has been directly measured ${ }^{7}$ ) and was found to be positive. From the values of $R$ and $A$ it is possible to obtain $\mathrm{O}_{3}$, which gives us again the product of the moduli as function of $\phi$ (see appendix 1):

$O_{3}=R \sin \theta_{\mathrm{R}}+A \cos \theta_{\mathrm{R}}=2\left|f_{++}\right|\left|f_{+-}\right| \cos \phi$.

Therefore $\rho$ must satisfy the equation

$\rho^{2}-\rho \sigma+\frac{O_{3}^{2}}{4 \cos ^{2} \phi}=0$.

In fig. 3 we plotted two curves, corresponding to eqs. (7a) and (10) respectively, which intersect each other at two points $\mathrm{B}$ and $\mathrm{C}$. This gives two possible sets of solutions for the moduli of the amplitudes. However, since $A$ has been measured to be positive, we can only keep the solution $\left|f_{++}\right|>\left|f_{+-}\right|$(solution B). We find numerically, if we normalize in such a way that $\sigma=1$

$$
\begin{aligned}
\left|f_{++}\right| & =0.996 \pm 0.006 \\
\left|f_{+-}\right| & =0.09 \pm 0.06 \\
\phi & =-37^{\circ} \pm 20^{\circ} .
\end{aligned}
$$

* Note that $\sin \phi$ has to be negative, since $P>0$ (see app. 1). 
This phase $\phi$ is badly determined, because $P$ and $O_{3}$ happen to have almost the same magnitude, which makes the two curves overlapping for a large range of $\phi$, if the errors are included, This example illustrates, that the accuracy of the final amplitudes can depend very much on the relative magnitude of the observables.

\section{NN elastic scattering}

For a review on the formalism of nucleon-nucleon scattering we refer the reader to the works by Hoshizaki ${ }^{8}$ ) and Moravcsik ${ }^{9}$ ).

Proton-proton elastic scattering can be described in terms of five complex amplitudes, if one assumes parity invariance, time reversal invariance and identical particle symmetry. Common sets of amplitudes are the standard s-channel helicity amplitudes ${ }^{10}$ ), the transversity amplitudes ${ }^{11}$ ) or the exchange amplitudes ${ }^{12}$ ). These sets are simple linear combinations of each other.

In order to simplify an analytic reconstruction of the amplitudes, we found it convenient to define a set of amplitudes $n_{1}, n_{2}, n_{3}, u_{1}$, and $u_{2}$, which are defined in terms of the standards-channel helicity amplitudes in appendix 2.1. Of course, an analytic reconstruction can be done with different sets of amplitudes as well, but the expressions for the laboratory observables of interest become more complicated. Our set of amplitudes is closely related to the transversity amplitudes ${ }^{11}$ ). The amplitudes $u_{1}$ and $u_{2}$ are the double spin flip amplitudes, corresponding to unnatural parity exchange, while $n_{1}, n_{2}$, and $n_{3}$ are simple combinations of the non-flip amplitudes, corresponding to natural parity exchange.

The amplitudes $u_{1}$ and $u_{2}$ are expected to be small, while $n_{1}$ and $n_{2}$ are expected to be the largest ones, having similar magnitudes since their difference is proportional to the double helicity flip amplitudes (see appendix 2.1). The amplitude $n_{3}$, corresponding to single helicity flip, is expected to be smaller than the non-flip ones. Therefore we expect to first order (at least for small $t$-values)

$u_{1} \approx u_{2}<n_{3}<n_{1} \approx n_{2}$.

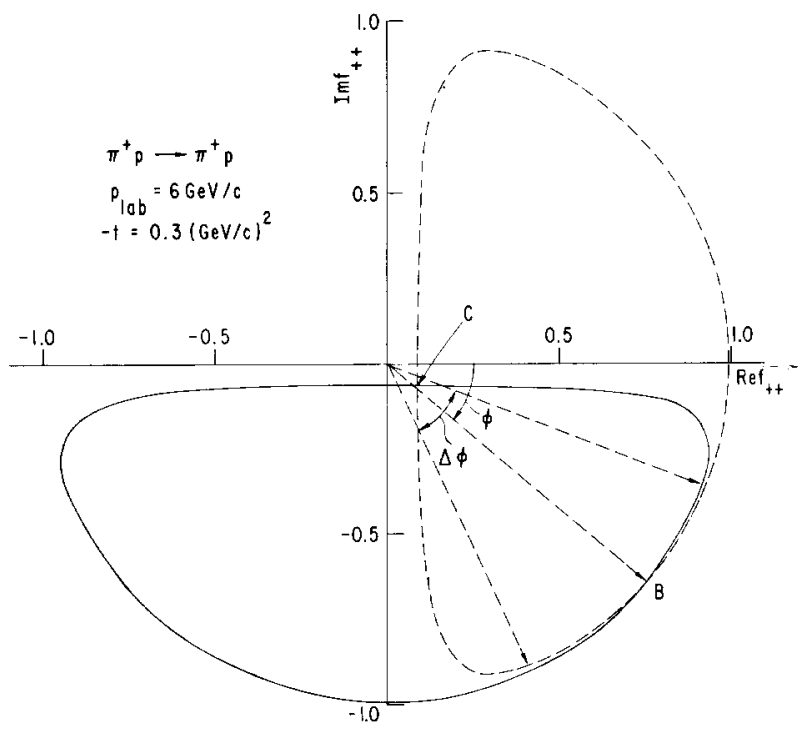

Fig. 3. The two curves, corresponding to eqs. (7a) and (10) in the text, intersect each other at two points $B$ and $C$, which gives two possible solutions for $f_{++}$.
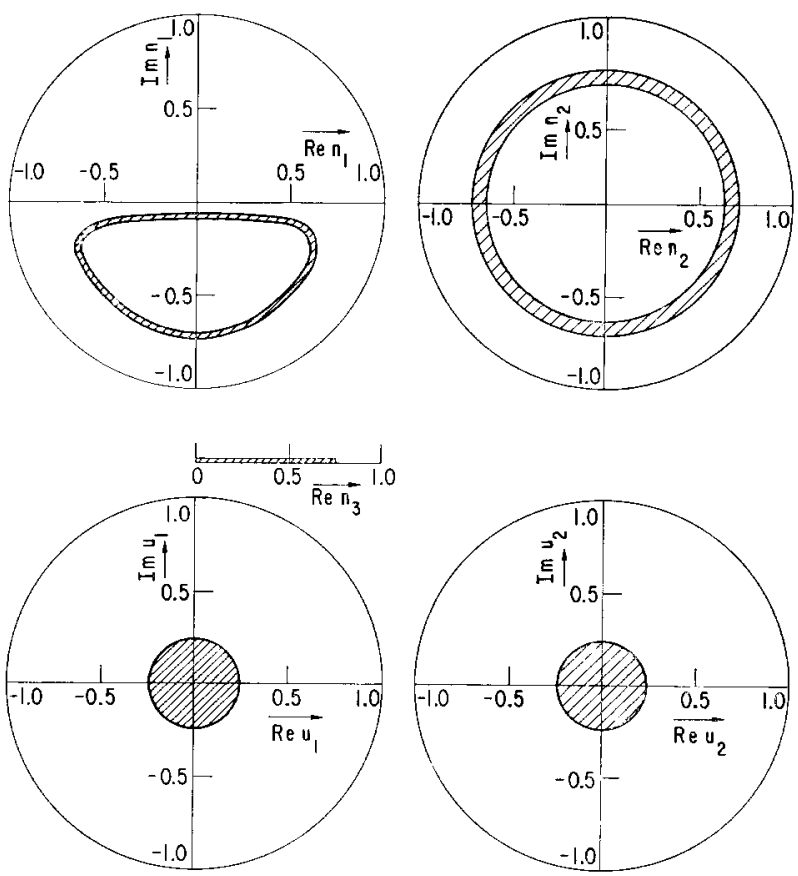

Fig. 4. Restrictions on the five pp elastic scattering amplitudes, imposed by measurement of the differential cross section and the Wolfenstein parameters $P, D_{N N}, K_{N N}$ and $C_{N N}$. 
An estimate of $\left|u_{1}\right|$ and $\left|u_{2}\right|$ can be obtained from the values of the Wolfenstein parameters ${ }^{13}$ ) $D_{N N}, K_{N N}$ and $C_{N N}$, since [see eqs. (15) and (16) hereafter]

$\left|u_{1}\right|^{2}+\left|u_{2}\right|^{2}=\frac{1}{2}\left(1-D_{N N}\right)$,

and also

$\left|u_{1}\right|^{2}-\left|u_{2}\right|^{2}=\frac{1}{2}\left(K_{N N}-C_{N N}\right)$,

where $D_{N N} \approx 1{ }^{14}$ ) and $K_{N N} \approx C_{N N}{ }^{15}$ ) at $6 \mathrm{GeV} / c$ and low transverse momenta. In case of five amplitudes one can perform 25 independent measurements. In appendix 2.2 we have written the 25 center-of-mass (c.m.) observables of ref. 12 in terms of our set of amplitudes. Linear relations between these observables and other c.m. observables can be obtained from table 2 in ref. 12 . In appendix 2.3 we give the $M$-matrix, from which the 16 quadratic relations corresponding to eqs. (3) and (4) can be obtained. The laboratory observables in the $s, n, l$ coordinate system of ing. 1 can be obtained from the c.m. observables by simple rotations ${ }^{12}$ ). Due to this rotation a lab observable an become a linear combination of two c.m. observables. In that case we denoted this c.m. observable as an ndirect measurement in appendix 2.2 .

The experimental program on the way at the ZGS at Argonne National Laboratory allows 15 different measurenents, if the following, technically more difficult, measurements are avoided: (1) the spin of the fast forward article is not analyzed, (2) the beam is not polarized longitudinally, (3) the recoil spin is not analyzed in the lontudinal direction. This set of technically more difficult measurements has been denoted by I instead of I in appendix 2.2. In appendix 2.4 we have listed the remaining 15 laboratory observables in terms of the center-of-mass observables. In appendix 2.5 we give quantitative estimates for them from the amplitudes given in ref. 16. For comparison we give also the existing data on 6 of the measured observables, Now we will describe, how one can reconstruct the $s$-channel helicity amplitudes from a suitable set of 11 laboratory observables, of which 6 have been measured already. We will assume an experimental error of 0.08 for all the unknown double scattering experiments. The calculations are done in 3 stages. First we calculate, how much the present data restricts the amplitudes. Then we calculate the restrictions imposed by $R$ and $A$ type measurements. At the end we will discuss the determination of the remaining unknown phase.

\subsection{Restrictions on the Amplitudes After five DiReCt MeASUREMENTS $\sigma, P, D_{N N}, C_{N N}$ AND $K_{N N}$}

These so-called " $N$-measurements" have already been performed by different experimental groups ${ }^{14,15,17,18}$ ), and it is relevant to ask, how much of the amplitudes do we know at this stage. From appendix 2.2 we find immediately

$\left.\left|n_{2}\right|=\frac{1}{2} \sqrt{(} I_{1}+I_{5}+I_{9}+I_{11}\right)$,

$\left|u_{1}\right|=\frac{1}{2} \sqrt{ }\left(I_{1}-I_{5}-I_{11}+I_{9}\right)$,

$\left|u_{2}\right|=\frac{1}{2} \sqrt{ }\left(I_{1}-I_{5}+I_{11}-I_{9}\right)$,

$S \equiv\left|n_{1}\right|^{2}+\left|n_{3}\right|^{2}=\frac{1}{4}\left(I_{1}+I_{5}-I_{9}-I_{11}\right)$,

$\left|n_{1}\right|^{2}\left|n_{3}\right|^{2}=\frac{I_{2}^{2}}{4 \sin ^{2} \phi_{1}}$

We recall, that $\sigma=I_{1}, P=-I_{2}, D_{N N}=I_{5}, C_{N N}=-I_{9}$, and $K_{N N}=-I_{11}$.

Since we can choose one phase arbitrarily, we assume $n_{3}$ real positive and $\phi_{1}$ is the relative phase between $n_{1}$ and $n_{3}$. Eqs. (14)-(16) give the moduli $n_{2}, u_{\text {: }}$, and $u_{2}$. Eqs. (17) and (18) can be used in the same way as in $\pi N$ scattering to give a relation between $\left|n_{1}\right|,\left|n_{3}\right|$, and $\phi_{1}$. The analogue of eq. (7a) is

$\rho^{2}-S \rho+\frac{I_{2}^{2}}{4 \sin ^{2} \phi_{1}}=0$ 
and to get $\rho$ real, we must have

$S^{2}>\frac{I_{2}^{2}}{\sin ^{2} \phi_{1}}$.

Eq. (20) gives us a range for $\phi_{1}$ and the following restriction

$|S|>\left|I_{2}\right|$,

which can be written as

$\left|\sigma+D_{N N}+C_{N N}+K_{N N}\right|>4|P|$.

This non-trivial inequality is satisfied by the present data. Eq. ( $21 \mathrm{~b})$ is a special case of eq. (5), that is

$\operatorname{Im} M_{15} \leqslant \sqrt{ } M_{11} \sqrt{ } M_{55}$.

Using the numerical values of appendix 2.5, we obtain

$\left|n_{2}\right|=0.68 \pm 0.04$,

$\left|u_{1}\right|=0.01 \pm 0.21$,

$\left|u_{2}\right|=0.01 \pm 0.21$,

$S=0.53 \pm 0.03$,

$-168^{\circ}<\phi_{1}<-12^{\circ}$.

Fig. 4 summarizes the results after the five measurements. We have some constraints on the moduli, but the phases are almost totally unknown.

In order to obtain the phases, one has to perform new measurements. Suppose one measures the $R$ and $A$ parameters, corresponding to $I(\mathrm{o} s ; o s)$ and $I(\mathrm{o} / ; \mathrm{os})$, respectively, and using also a polarized beam, so that $I(n s ; o s)$ and $I(n l ; o s)$ are measured simultaneously. Then one can obtain all the amplitudes, except for one phase, as will be shown below.

\subsection{ReStRictions on the AMPLITUDES IMPOSED By $R$ AND $A$ TYPE MEASUREMENTS}

Let us assume, that the measurements $R=I(\mathrm{os} ; \mathrm{os})$ and $A=I(\mathrm{o} l ; o s)$, and simultaneously $I(n s ; o s)$ and $I(n l ; o s)$ have been performed and let us consider the following four quantities

$l_{1}=I(\mathrm{o} l ; \mathrm{os}) \cos \theta_{\mathrm{R}}-I(\mathrm{os} ; \mathrm{os}) \sin \theta_{\mathrm{R}}$,

$l_{2}=I(\mathrm{o} l ; \mathrm{os}) \cos \theta_{\mathrm{R}}+I(\mathrm{os} ; \mathrm{os}) \sin \theta_{\mathrm{R}}$,

$l_{1}^{\prime}=I(n s ; o s) \cos \theta_{\mathrm{R}}+I(n l ; o s) \sin \theta_{\mathrm{R}}$,

$l_{2}^{\prime}=I(n s ; o s) \cos \theta_{\mathrm{R}}-I(n l ; o s) \sin \theta_{\mathrm{R}}$,

where $\theta_{\mathbf{R}}$ is again the lab recoil angle. We can define two complex numbers $L_{1}$ and $L_{2}$ as follows

$L_{1}=l_{1}+\mathrm{i} l_{1}^{\prime}$,

$L_{2}=l_{2}+\mathrm{i} l_{2}^{\prime}$,

and after a straightforward calculation, using appendices 2.2 and 2.4 we get

$L_{1}=\left(\alpha n_{1}-\beta n_{3}\right) n_{2}^{*}$,

$L_{2}=-2 n_{3} n_{2}^{*}+\alpha u_{1}^{*} u_{2}$,

where

$\alpha=2 \sin 2 \theta_{\mathrm{R}}, \quad$ and $\quad \beta=2 \cos 2 \theta_{\mathrm{R}}$. 
Here $L_{1}$ and $L_{2}$ are known from the measurements, while also the moduli of all the amplitudes are known within some errors. Thus from eq. (25) one can obtain the phases of the natural parity exchange amplitudes $n_{1}$ and $n_{2}$, which we called $\phi_{1}$ and $\phi_{2}$, respectively. Recall that the phase of $n_{3}$ is assumed to be zero. From eq. (26) one can obtain the relative phase between the unnatural parity exchange amplitudes. Let us concentrate on $L_{1}$. At this stage we know

$\left|n_{2}\right|$,

$S \equiv\left|n_{1}\right|^{2}+\left|n_{3}\right|^{2}$,

and

$\left|n_{1}\right|^{2}\left|n_{3}\right|^{2}=I_{2}^{2} / 4 \sin ^{2} \phi_{1}$.

Eq. (25) can be rewritten as

$L_{1}=\alpha\left|n_{1}\right|\left|n_{2}\right| \mathrm{e}^{\mathrm{i}\left(\phi_{1}-\phi_{2}\right)}-\beta\left|n_{2}\right|\left|n_{3}\right| \mathrm{e}^{-\mathrm{i} \phi_{2}}$,

from which follows

$\left|L_{1}\right|^{2}=\left|n_{2}\right|^{2}\left[\alpha^{2}\left|n_{1}\right|^{2}+\beta^{2}\left|n_{3}\right|^{2}-2 \alpha \beta\left|n_{1}\right|\left|n_{3}\right| \cos \phi_{1}\right]$.

We can eliminate $\phi_{1}$ by calculating $\cos ^{2} \phi_{1}$, and adding $\sin ^{2} \phi_{1}$ to it. Then we obtain

$4\left|n_{1}\right|^{2}\left|n_{3}\right|^{2}=I_{2}^{2}+\left[\frac{\left|L_{1}\right|^{2}}{\alpha \beta\left|n_{2}\right|^{2}}-\frac{\beta}{\alpha} S+\frac{\beta^{2}-\alpha^{2}}{\alpha \beta}\left|n_{1}\right|^{2}\right]^{2}$,

We can also elminate $\left|n_{3}\right|^{2}$ by using

$\left|n_{3}\right|^{2}=S-\left|n_{1}\right|^{2}$,

and after some easy algebra we obtain a simple quadratic equation for $\rho=\left|n_{1}\right|^{2}$ :

$16 \rho^{2}+2\left[M\left(\beta^{2}-\alpha^{2}\right)-4 \beta^{2} S\right] \rho+\alpha^{2} \beta^{2} I_{2}^{2}+\left[M-\beta^{2} S\right]^{2}=0$

Here $M$ is defined as $M \equiv\left|L_{1}\right|^{2} /\left|n_{2}\right|^{2}$.

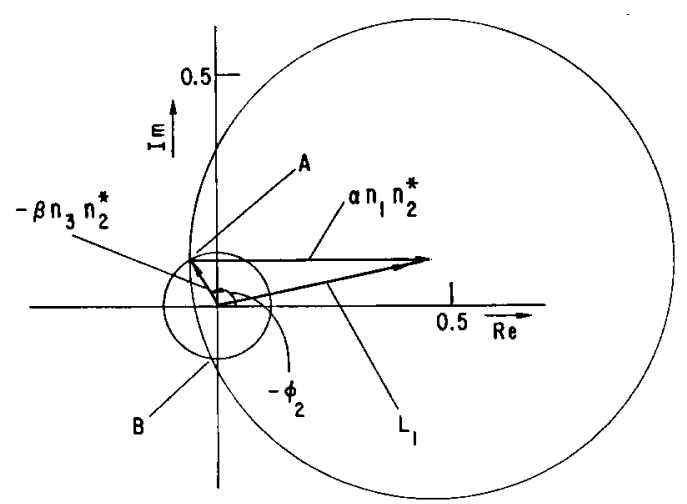

Fig. 5a. Determination of the phases of the natural parity exchange amplitudes by constructing $L_{1}=\alpha n_{1} n_{2}^{*}-\beta n_{3} n_{2}^{*}$. Here $\left|n_{1}\right|$ was taken to be $\rho_{+}=0.72 \pm 0.03$, which gives two possible solutions $\mathbf{A}$ and $\mathbf{B}$. The phase of $n_{3}$ is assumed to be 0 . The phase of $n_{2}$ is given by the angle $-\phi_{2}$, while the phase difference between $n_{1}$ and $n_{2}, \phi_{1}-\phi_{2}$, is given by the direction of $\alpha n_{1} n_{2}^{*}$ $\left(\phi_{1}-\phi_{2}=0^{\circ}\right)$. The solution, corresponding to point $\mathrm{B}$, has to be rejected, because $\phi_{1}$ has to be negative, since the polarization $F \propto-\sin \phi_{1}$ is positive.

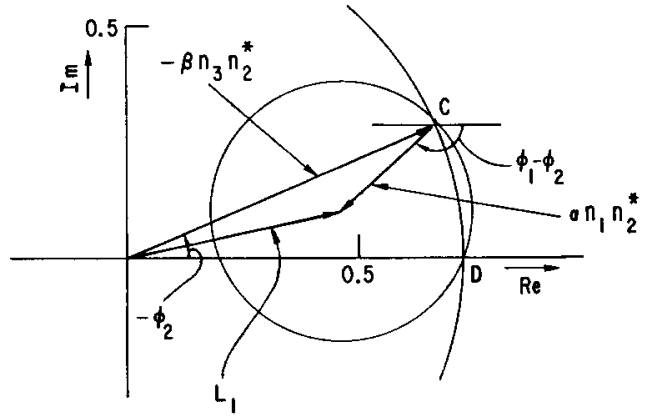

Fig. 5b. See fig. 5a, except $\left|n_{1}\right|$ was taken to be $\rho_{-}=0.37 \pm 0.03$. We find again two solutions (C and D), namely $n_{1}=0.37 \mathrm{e}^{ \pm \mathbf{i}\left(162^{\circ}\right)}$. However, these solutions do not agree with the range for $n_{1}$, given in fig. 4 , and have to be rejected therefore. 
The solutions of this equation are given by

$\rho_{ \pm}=\frac{1}{2}\left\{S+\left(S-\frac{1}{2} M\right) \cos \left(4 \theta_{\mathrm{R}}\right) \pm \sqrt{ }\left[\left(S-\frac{1}{4} M\right) M-I_{2}^{2}\right] \sin \left(4 \theta_{\mathrm{R}}\right)\right\}$.

Thus we have an ambiguity in $\left|n_{1}\right|^{2}$, since it can be $\rho_{+}$or $\rho_{-}$. Then we have also an ambiguity in $\left|n_{3}\right|$, because of eq. (30). These ambiguities can be solved by calculating the phases for each solution. The phases $\phi_{1}$ and $\phi_{2}$ can be obtained from eq. (27) via a geometrical method. One just has to construct the known complex number $L_{1}$, draw a circle of radius $r_{1}=\alpha\left|n_{1}\right|\left|n_{2}\right|$ centered in $L_{1}$, and another circle of radius $r_{2}=\beta\left|n_{2}\right|\left|n_{3}\right|$ centered at the origin. In general the two circles will intersect in two points, provide the solutions of eq. (3I) are real and positive. This requires

$\left(S-\frac{1}{4} M\right) M \geqslant I_{2}^{2}$,

which gives the inequalities

$\left.2\left|n_{2}\right|^{2}\left[S-\sqrt{ }\left(S-I_{2}^{2}\right)\right] \leqslant\left|L_{1}\right|^{2}<2\left|n_{2}\right|^{2}\left[S+\sqrt{ } S-I_{2}^{2}\right)\right]$.

These inequalities, which give bounds on the possible values of $\left|L_{1}\right|^{2}$, are a special case of eq. (5).

The geometrical method is illustrated in figs. $5 \mathrm{a}$ and $5 \mathrm{~b}$, using the numerical values for the observables of appendix 2.5. Fig. 5a corresponds to $\rho_{+}$, which gives $\left|n_{1}\right|=0.72 \pm 0.03$, and fig. $5 \mathrm{~b}$ to $\rho_{-}$, which gives $\left|n_{1}\right|=0.37 \pm 0.03$. Each figure gives two solutions for the phases $\phi_{1}$ and $\phi_{2}$, so we have in total a four-fold ambiguity in $n_{1}$ corresponding to the points $\mathrm{A}, \mathrm{B}, \mathrm{C}$, and $\mathrm{D}$ in fig. 5. The points $\mathrm{A}$ and $\mathrm{C}$ would give the solutions

$n_{1}=0.72 \mathrm{e}^{-\mathrm{i}\left(122^{\circ}\right)}$,

and

$n_{1}=0.37 \mathrm{e}^{-\mathrm{i}\left(162^{\circ}\right)}$,

while the points $\mathrm{B}$ and $\mathrm{D}$ would give the same solutions with an opposite sign for the phase of $n_{1}$. However, the latter solutions are not allowed by the range for $\phi_{1}$ given in eq. (22). If the other solutions for $n_{1}$ are compared with the solution of eq. (19), which has been given graphically in fig. 4, then it turns out that one can keep only the solution

$n_{1}=(0.72 \pm 0.03) \mathrm{e}^{-\mathrm{i}\left(122^{\circ} \pm 32^{\circ}\right)}$

The quoted error on the phase is just a global estimate, since it was obtained from the range allowed by the circles with minimum and maximum radii. The errors on the phases quoted hereafter will be obtained in the same way. From fig. 5 we also obtain the phase of $n_{2}$, which gives

$n_{2}=(0.68 \pm 0.04) \mathrm{e}^{-\mathrm{i}\left(122^{\circ} \pm 30^{\circ}\right)}$.

Since $n_{1}$ is pinned down, we find immeditely $n_{3}$ to be

$n_{3}=\left(0.10_{-0.02}^{+0} 06\right)$,

because of the restrictions imposed by eqs. (17)-(19), which give one-to-one relationship between $n_{1}$ and $n_{3}$, as explained in fig. 2 for the case of $\pi N$ scattering.

Since $\phi_{2}$ is now determined, we can use eq. (26) to obtain the relative phase between $u_{1}$ and $u_{2}$. A solution of eq. (26) is given in fig. 6 , where we constructed $L_{2}$ and the range for $2 n_{3} n_{2}^{*}\left(\Delta \phi_{2}= \pm 32^{\circ}\right)$. This gives us the following range for the phase difference $\phi_{34}$ between $u_{1}$ and $u_{2}$ :

$-225^{\circ}<\phi_{34}<-15^{\circ}$.

Here we did not take the experimental error on $L_{2}$ into account. However, in order to obtain some information on the phase of $\alpha u_{1}^{*} u_{2}$, one has to measure $\left\{\begin{array}{l}\mathrm{Re} \\ \mathrm{Im}\end{array}\right\} L_{2}$ with an accuracy of the order of $\alpha\left|u_{1}\right|\left|u_{2}\right|$, which is $\sim 10^{-4}$ in our specific example. Therefore with our assumed error of 0.08 we obtain no information on $\phi_{34}$. We will discuss this later on. Our knowledge about the amplitudes at this point has been summarized in fig. 8 . We will now discuss a way to determine the phases of the unnatural parity exchange amplitudes. We will not take the 
experimental errors into account, since in our specific example the moduli of $u_{1}$ and $u_{2}$ are too small to obtain these amplitudes accurately from the measurements. Fortunately. if they are really so small in practice, then there is no need to determine them accurately, since they will be negligible as compared to the other amplitudes. However, at large transverse momenta the unnatural parity amplitudes may play an appreciable role. Then one should be able to obtain them from the previous experiments in combination with the experiments, mentioned below.

\subsection{Restrictions on the AMPLitudes imposed by the MEASUREMENTS I ( $s o ;$ os) AND I (sn;os)}

Let us assume, that $I(s o ; o s)$ and $I(s n ; o s)$ have been measured and consider the complex number $L_{3}=I(s o ; o s) \cos \theta_{\mathrm{R}}-\mathrm{i} I(s n ; o s) \sin \theta_{\mathrm{R}}$.

By using appendices 2.2 and 2.4 one can easily show that

$l_{3}=-\frac{1}{2} \alpha u_{1} n_{3}^{*}+n_{2} u_{2}^{*}-n_{1} u_{1}^{*}+\frac{1}{2} \beta\left(n_{2}^{*} u_{2}-n_{1}^{*} u_{1}\right)$,

which can be rewritten as

$L_{3}=\zeta_{1} \mathrm{e}^{\mathrm{i} \phi_{3}}+\zeta_{2} \mathrm{e}^{-\mathrm{i} \phi_{3}}$,

with

$\zeta_{1}=-\frac{1}{2} \alpha\left|u_{1}\right|\left|n_{3}\right|+\frac{1}{2} \beta\left[\left|n_{2}\right|\left|u_{2}\right| \mathrm{e}^{-\mathrm{i}\left(\phi_{2}+\phi_{34}\right)}+\left|n_{1}\right|\left|u_{1}\right| \mathrm{e}^{-\mathrm{i}\left(\pi+\phi_{1}\right)}\right]$,

$\zeta_{2}=\left|n_{2}\right|\left|u_{2}\right| \mathrm{e}^{\mathrm{i}\left(\phi_{2}+\phi_{34}\right)}+\left|n_{1}\right|\left|u_{1}\right| \mathrm{e}^{\mathrm{i}\left(\pi+\phi_{1}\right)}$.

Here $L_{3}, \zeta_{1}$, and $\xi_{2}$ are known and one can solve the equation via the geometrical method (see fig. 7), which gives for the phase of $u_{1}$

$\phi_{3}=145^{\circ}$,

and therefore we find the phase of $u_{2}$ to be

$\phi_{4}=325^{\circ}$,

since their phase difference $\phi_{34}$ was determined to be $-180^{\circ}$ (from fig. 6 ), if we neglect the experimental errors. Even if $L_{3}$ is known accurately, then the large error in $\phi_{34}$ leaves $\phi_{3}$ and $\phi_{4}$ undetermined.

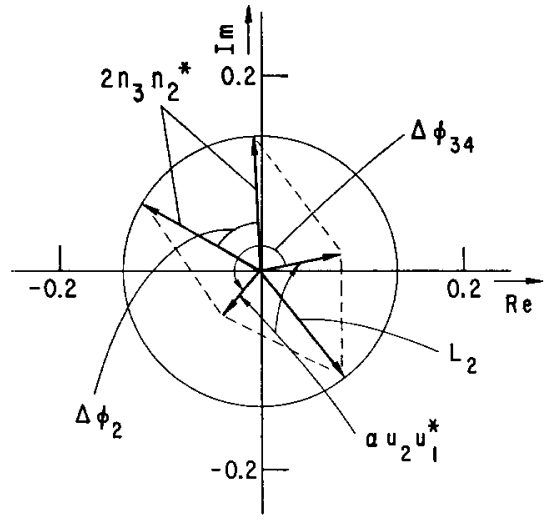

Fig. 6. Determination of the phase difference $\phi_{34}$ between the two unnatural parity exchange amplitudes by constructing $L_{2}=-2 n_{3} n_{2}^{*}+\alpha u_{2} u_{1}^{*} . \Delta \phi_{2}$ indicates the error on $\phi_{2}$, which causes a large error $\Delta \phi_{34}$ in $\phi_{34}$. If, in addition an error of 0.08 for $\left|L_{2}\right|$ is taken into account, then one finds no constraints on $\phi_{34}$.

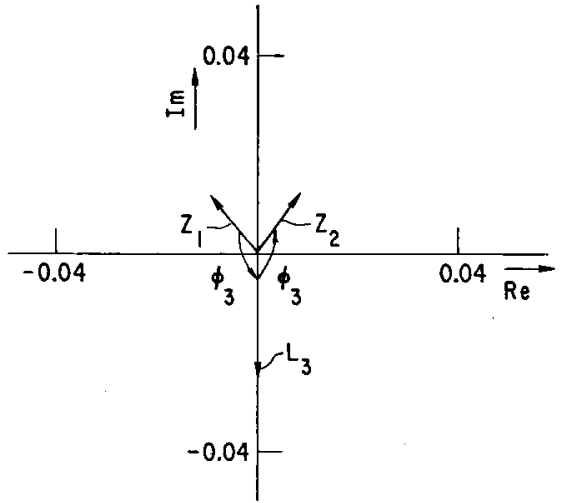

Fig. 7. Determination of the phase $\phi_{3}$ of the unnatural parity exchange amplitude $u_{1}$ by construction of $L_{3}=\left|\zeta_{1}\right| \mathrm{e}^{1 \phi_{3}}+$ $+\left|\zeta_{2}\right| \mathrm{e}^{-1 \phi_{3}}$. If typical experimental errors are taken into account, one finds no constraints on $\phi_{3}$. 

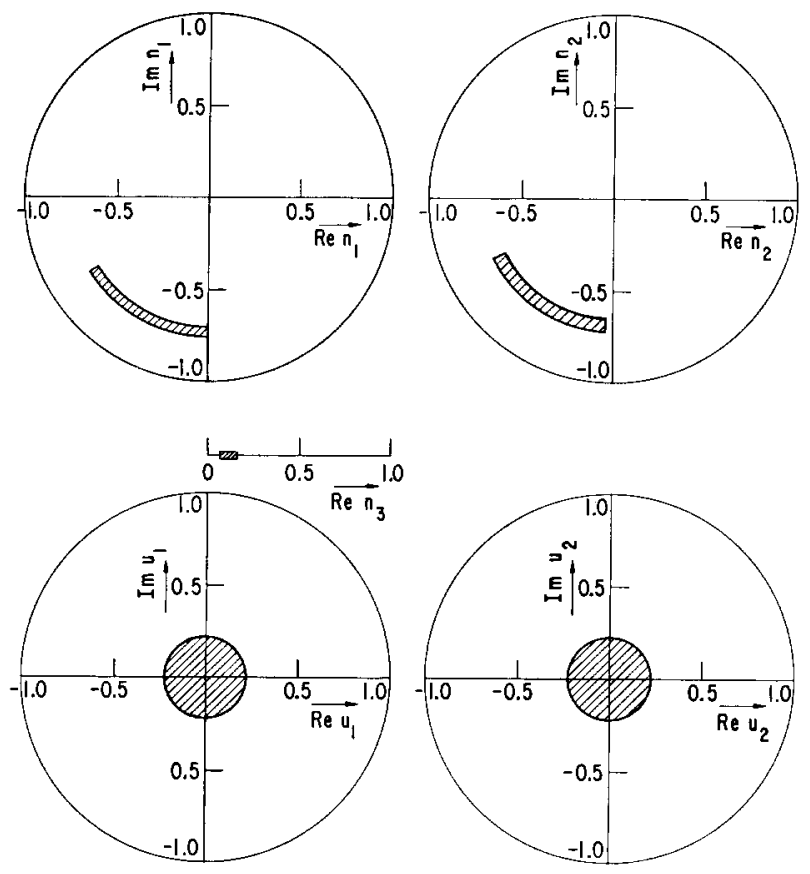

Fig. 8. Summary of the knowledge of the pp elastic scattering amplitudes after 9 suitable measurements, namely the 5 measurements which determine fig. 4 , and $I(\mathrm{os} ; \mathrm{o} s), I(n s ; \mathrm{o} s), I(\mathrm{o} l ; \mathrm{o} s)$, and $I(n l ; o s)$.

\section{Discussion}

The advent of a high energy polarized beam has made it feasible to perform sufficient experiments for the reconstruction of the five pp elastic scattering amplitudes.

We found an unambiguous solution for the amplitudes, if in addition to the performed or recently proposed measurements, $I(\mathrm{oo} ; \mathrm{oo}), I(\mathrm{o} n ; \mathrm{oo}), I(n n ; \mathrm{oo}), I(\mathrm{o} n ; \mathrm{o} n), I(n o ; \mathrm{o} n), I(s o ; \mathrm{os})$, and $I(s n ; o s)$, one knows $I(\mathrm{os} ; \mathrm{os})$, $I(n s ; \mathrm{os}), I(\mathrm{o} l ; \mathrm{os})$, and $I(n l ; \mathrm{os})$.

These last four observables require only two experiments, if one uses a polarized beam in combination with a polarized target and a recoil polarimeter. We solved the equations for a specific numerical example, taking into account the experimental errors. An appreciable contribution to the uncertainties in the phases comes from the uncertainties in the moduli, which are mainly determined by the errors on $D_{N N}$ and $K_{N N}$ (see figs. 5 and 6).

In appendix 2.4 we listed the lab observables, which are the easiest to measure. However, if $D_{N N} \approx 1$, then $\left|u_{1}\right| \approx\left|u_{2}\right| \approx 0$ [see eq. (12)]. In that case, six of these observables are zero (see table 1). Therefore it will be difficult to obtain new information from these experiments at small $t$-values, where $D_{N N} \approx 1^{14}$ ). Fortunately, these observables give then information on negligible amplitudes. However, at large $t$-values $u_{1}$ and $u_{2}$ are not negligible, and at least two of the observables of table 1 have to be known to obtain the phases of $u_{1}$ and $u_{2}$.

In the previous sections we assumed that we measured the laboratory observables of appendix 2.4 pure, that

Table 1

Some of the lab observables of appendix 2.4. These observables are zero, if the unnatural parity exchange amplitudes are zero, which is the case if $D_{N N}=1$. From these observables one can obtain the phases of the unnatural parity exchange amplitudes, if $1-D_{N N}$ differs appreciably from one, which is the case at large $t$-values.

$\begin{array}{lll}I(s l ; 0 o) & I(s s ; o n) & I(s \circ ; \circ s) \\ I(s s ; \circ) & I(s l ; 0 n) & I(s n ; o s)\end{array}$


is only $s, l$ or $n$ components of the polarization were involved. However, in an experimental set-up, vertical magnetic fields are usually present which cause a precession of the horizontal spin components. Instead of measuring these components "pure", one measures linear combinations of them. In this case an analytic reconstruction of the amplitudes becomes more difficult. Then it may be more convenient to reconstruct the amplitudes via a numerical method ${ }^{21}$ ) by varying the amplitudes at random, calculating for each set the measured observables, and keeping only the solutions, which are consistent with the measurements.

However, the solution of the "simple" case, which we showed, is still very useful for obtaining insight in the various ambiguities and which experiments are important to resolve them.

We wish to thank Drs G. Thomas and C. Ward for useful comments and Dr R. Field for communicating results prior to publication. One of us (J.S.) acknowledges the warm hospitality received at Argonne National Laboratory.

\section{References}

1) M. Simonius, Phys. Rev. Lett. 19 (1967) 279; G. R. Goldstein, M. J. Moravcsik and D. Bregman, Lett. Nuovo Cimento, 11 (1974) 137; M. Mac Gregor, M. Moravcsik and H. Stapp, Ann. Rev. Nucl. Sci. 10 (1960) 291.

$\left.{ }^{2}\right)$ C. Schumacher and H. Bethe, Phys. Rev. 121 (1961) 1534; S. M. Bilenky, L. I. Lapidus and R. M. Ryndin, Sov. Phys. JETP 24 (1966) 593; G. H. Thomas, Thesis (UCLA 1969); N. Byers and C. N. Yang, Phys. Rev. $135 B$ (1964) 796.

3) J. T. Donohue, Phys. Rev. D11 (1975) 1935; M. Doncel, L. Michel and P. Minnaert, CERN preprint D. Ph. II/PHYS 74-7.

4) A. Yokosawa et al., ZGS proposal E-385.

5) C. Bourrely and J. Soffer, Phys. Rev. D12 (1975) 2932; R. M. Delaney and J. L. Gammel, Phys. Rev. D12 (1975) 1978.

6) M. Borghini et al., Phys. Lett. $31 B$ (1970) 405.

7) A. de Lesquen et al., Phys. Lett. $40 B(1972) 278$.

8) N. Hoshizaki, Supp. Prog. Theor. Phys. 42 (1968) 107.

$\left.{ }^{9}\right)$ M. J. Moravcsik, The nucleon-nucleon interaction (Clarendon Press, Oxford, 1963).

10) M. Jacob and G. C. Wick, Ann. Phys. 7 (1959) 404.

11) J. T. Donohue, Proc. Summer Studies on High energy physics with polarized beams (ed. J. B. Roberts; ANL-HEP 75-02) p. I-1.

12) F. Halzen and G. H. Thomas, Phys. Rev. D10 (1974) 344.

13) L. Wolfenstein, Ann. Rev. Nucl. Sci. 6 (1956) 43.

14) G. W. Abshire et al., Phys. Rev. D12 (1975) 3393.

${ }^{15)}$ R. C. Fernow et al., Phys. Lett. $52 B$ (1974) 243.

${ }^{16}$ ) C. Bourrely, A. Martin, J. Soffer and D. Wray, preprint ANL-HEP-PR 75-41 (Aug. 1975).

17) M. Borghini et al., Phys. Lett. 36B (1971) 501.

18) G. Hicks et al., Phys. Rev. D12 (1975) 2594.

19) One pair of possible observables to determine the phases of the unnatural parity exchange amplitudes has been given in Section 4.3 . Here the phase of $u_{1}$ was determined from the interference term between two small amplitudes $u_{1}$ and $u_{2}$. One can try to determine the phases of $u_{1}$ and $u_{2}$ from an interference term with a large amplitude, which may be easier to observe. For example, one can try to measure $I(s s ; 0 o)$ and $I(s l ; o n)$, which involve both the real and imaginary parts of $n_{2} u_{1}^{*}$ and $n_{1} u_{2}^{*}$. To obtain the phases of $u_{1}$ and $u_{2}$, one can again apply the geometrical method by constructing the complex number $Z=I(s s ; 00)+\mathrm{i} I(s l ;$ on $)$. However, in our specific example, these observables are also small, because two large terms tend to cancel one another and one does not gain very much as compared to the observables in section 4.3 .

20) J. Deregel et al., Phys. Lett. $43 B$ (1973) 338.

21) G. Thomas, private communication.

22) R. Field, private communication.

\section{Appendix 1}

$\pi N$ elastic scattering

1.1. The center-of-mass observables.

$o_{1}=\left|f_{++}\right|^{2}+\left|f_{+--}\right|^{2}$,

$o_{2}=2 \operatorname{Im}\left(f_{++} f_{+-}^{*}\right)$,

$o_{3}=2 \operatorname{Re}\left(f_{++} f_{+-}^{*}\right)$,

$O_{4}=\left|f_{++}\right|^{2}-\left|f_{+--}\right|^{2}$. 
1.2. The laboratory observables $\left(\theta_{\mathrm{R}}\right.$ is the lab recoil angle).

$\sigma=O(0 ; 0)=O_{1}$,

$P=O(n ; \mathrm{o})=-\mathrm{O}_{2}$,

$R=O(s ; s)=-O_{4} \cos \theta_{\mathrm{R}}-O_{3} \sin \theta_{\mathrm{R}}$,

$A=O(l ; s)=O_{4} \sin \theta_{\mathrm{R}}-O_{3} \cos \theta_{\mathrm{R}}$.

1.3. The $M$ matrix in terms of the center-of-mass observables.

$\mathbf{M}=2 f_{i j} f_{i j}^{*}=\left(\begin{array}{ll}O_{1}+O_{4} & O_{3}+\mathrm{i} O_{2} \\ O_{3}-\mathrm{i} O_{2} & O_{1}-O_{4}\end{array}\right)$

1.4. The quadratic relation, which follows from eq. (3) in the text.

$\left(O_{1}+O_{4}\right)\left(O_{1}-O_{4}\right)=O_{3}^{2}+O_{2}^{2}$.

This relation can be written as

$P^{2}+R^{2}+A^{2}=\sigma^{2}$.

\section{Appendix 2}

$N N$ elastic scattering

2.1. Our set of amplitudes in terms of the s-channel helicity amplitudes.

$n_{1}=\frac{1}{2 \sqrt{ } 2}\left(\phi_{1}+\phi_{2}+\phi_{3}-\phi_{4}\right)$

$n_{2}=\frac{1}{2 \sqrt{ } 2}\left(\phi_{1}-\phi_{2}+\phi_{3}+\phi_{4}\right)$,

$n_{3}=\sqrt{ } 2 \phi_{5}$,

$u_{1}=\frac{1}{2 \sqrt{ } 2}\left(-\phi_{1}+\phi_{2}+\phi_{3}+\phi_{4}\right)$,

$u_{2}=\frac{1}{2 \sqrt{ } 2}\left(-\phi_{1}-\phi_{2}+\phi_{3}-\phi_{4}\right)$.

Here we used the standard notation for the helicity amplitudes:

$\phi_{1}=\langle++|\mathbf{M}|++\rangle$,

$\phi_{2}=\langle++|\mathbf{M}|--\rangle$,

$\phi_{3}=\langle+-|\mathbf{M}|+-\rangle$,

$\phi_{4}=\langle+-|\mathbf{M}|-+\rangle$,

$\phi_{5}=\langle++|\mathbf{M}|-+\rangle$.

The relations between our set of amplitudes and other commonly used sets can be found by comparison with ref. 8 for the "classical" amplitudes 9 ), ref. 11 for the transversity amplitudes and ref. 12 for the exchange amplitudes. 


\subsection{Center-of-mass observables.}

Below we list 25 independent c.m. observables in terms of the helicity amplitudes of an appendix 2.1. At the right hand side we give Halzen and Thomas' notation for the c.m. observables: $I(a b ; c d)$, where the arguments give the direction of the polarization of the beam, target, scattered and recoil particle, respectively. The c.m. observables can be expressed in terms of lab observables, which are defined in terms of polarization components along the coordinate axis of fig. 1. (see appendix 2.4). If the coordinate transformation causes a c.m. observable to be a linear combination of two lab observables, then it is denoted as an indirect measurement $(\tilde{I})$. The c.m. observables, which are difficult to measure, because of reasons given in section 4 , are denoted by $\bar{I}$.

$$
\begin{aligned}
& I_{1}=\left|n_{1}\right|^{2}+\left|n_{2}\right|^{2}+\left|n_{3}\right|^{2}+\left|u_{1}\right|^{2}+\left|u_{2}\right|^{2}, \quad I(\infty ; \infty) \text {, } \\
& I_{2}=2 \operatorname{Im}\left(n_{1} n_{3}^{*}\right), \quad I(\mathrm{o} y ; \infty \mathrm{o}), \\
& \tilde{I}_{3}=2 \operatorname{Re}\left(n_{1} n_{2}^{*}\right)+2 \operatorname{Re}\left(u_{1} u_{2}^{*}\right), \quad I(\mathrm{o} z ; \mathrm{o} z), \\
& \tilde{I}_{4}=-2 \operatorname{Re}\left(n_{2} n_{3}^{*}\right), \quad I(\mathrm{oz} ; \mathrm{ox}), \\
& I_{5}=\left|n_{1}\right|^{2}+\left|n_{2}\right|^{2}+\left|n_{3}\right|^{2}-\left|u_{1}\right|^{2}-\left|u_{2}\right|^{2}, \quad I(o y ; o y) \text {, } \\
& \tilde{I}_{6}=2 \operatorname{Re}\left(n_{1} n_{2}^{*}\right)-2 \operatorname{Re}\left(u_{1} u_{2}^{*}\right), \quad I(\mathrm{o} x ; \mathrm{o} x), \\
& \bar{I}_{7}=-2 \operatorname{Re}\left(n_{2} u_{1}^{*}\right)-2 \operatorname{Re}\left(n_{1} u_{2}^{*}\right), \quad I(z z ; \infty), \\
& I_{8}=2 \operatorname{Re}\left(u_{2} n_{3}^{*}\right), \quad I(x z ; \infty), \\
& I_{9}=-\left|n_{1}\right|^{2}+\left|n_{2}\right|^{2}-\left|n_{3}\right|^{2}+\left|u_{1}\right|^{2}-\left|u_{2}\right|^{2}, \quad I(y y ; o o), \\
& I_{10}=2 \operatorname{Re}\left(n_{2} u_{1}^{*}\right)-2 \operatorname{Re}\left(n_{1} u_{2}^{*}\right), \quad I(x x ; o \circ), \\
& I_{11}=-\left|n_{1}\right|^{2}+\left|n_{2}\right|^{2}-\left|n_{3}\right|^{2}-\left|u_{1}\right|^{2}+\left|u_{2}\right|^{2}, \quad I(y o ; o y), \\
& \tilde{I}_{12}=2 \operatorname{Re}\left(n_{1} u_{1}^{*}\right)-2 \operatorname{Re}\left(n_{2} u_{2}^{*}\right), \quad I(x \circ ; o x), \\
& \tilde{I}_{13}=2 \operatorname{Re}\left(u_{1} n_{3}^{*}\right), \quad I(x \circ ; 0 z), \\
& I_{14}=-2 \operatorname{Re}\left(n_{1} u_{1}^{*}\right)-2 \operatorname{Re}\left(n_{2} u_{2}^{*}\right), \quad I(\mathrm{o} z ; z 0), \\
& \tilde{I}_{15}=-2 \operatorname{Im}\left(n_{2} n_{3}^{*}\right), \quad I(y x ; o x), \\
& \tilde{I}_{16}=2 \operatorname{Im}\left(u_{1} n_{3}^{*}\right), \quad I(x y ; o x), \\
& I_{17}=-2 \operatorname{Im}\left(u_{2} n_{3}^{*}\right), \quad I(x x ; o y), \\
& \tilde{I}_{18}=2 \operatorname{Im}\left(u_{1} u_{2}^{*}\right)-2 \operatorname{Im}\left(n_{1} n_{2}^{*}\right), \quad I(y x ; \mathrm{oz}), \\
& \tilde{I}_{19}=2 \operatorname{Im}\left(n_{1} u_{1}^{*}\right)-2 \operatorname{Im}\left(n_{2} u_{2}^{*}\right), \quad I(x y ; \mathrm{o} z), \\
& \bar{I}_{20}=2 \operatorname{Im}\left(n_{1} u_{1}^{*}\right)+2 \operatorname{Im}\left(n_{2} u_{2}^{*}\right), \quad I(z y ; o x), \\
& \bar{I}_{21}=2 \operatorname{Im}\left(n_{1} u_{2}^{*}\right)+2 \operatorname{Im}\left(n_{2} u_{1}^{*}\right), \quad I(z x ; o y), \\
& \check{I}_{22}=2 \operatorname{Im}\left(u_{1} u_{2}^{*}\right)+2 \operatorname{Im}\left(n_{1} n_{2}^{*}\right), \quad I(y z ; o x), \\
& I_{23}=2 \operatorname{Im}\left(n_{1} u_{2}^{*}\right)+2 \operatorname{Im}\left(n_{2} u_{1}^{*}\right), \quad I(x z ; o y), \\
& \bar{I}_{24}=\left|n_{1}\right|^{2}+\left|n_{2}\right|^{2}-\left|n_{3}\right|^{2}+\left|u_{1}\right|^{2}+\left|u_{2}\right|^{2}, \quad I(x x ; x x) \text {, } \\
& \bar{I}_{25}=2 \operatorname{Re}\left(n_{1} n_{3}^{*}\right), \quad I(x x ; x z) .
\end{aligned}
$$


2.3. The $\mathbf{M}$ matrix in terms of the center-of-mass observables.

$\mathbf{M}=\left[\begin{array}{ccccc}-I_{9}-I_{11}+I_{5}+2 I_{24}-I_{1} & \tilde{I}_{3}+\tilde{I}_{6}+\mathrm{i}\left(\tilde{I}_{22}-\tilde{I}_{18}\right) & \tilde{I}_{12}-\tilde{I}_{14}+\mathrm{i}\left(\tilde{I}_{19}+\bar{I}_{20}\right) & -\tilde{I}_{7}-I_{10}+\mathrm{i}\left(\bar{I}_{21}-I_{23}\right) & 2 \bar{I}_{25}+2 \mathrm{i} I_{2} \\ & I_{1}+I_{5}+I_{11}+I_{9} & I_{10}-\bar{I}_{7}+\mathrm{i}\left(\tilde{I}_{2}+I_{23}\right) & -\tilde{I}_{12}-\bar{I}_{14}+\mathrm{i}\left(\bar{I}_{20}-\tilde{I}_{19}\right) & -2 \tilde{I}_{4}-2 \mathrm{i} \tilde{I}_{15} \\ & & I_{1}-I_{5}-I_{11}+I_{9} & \tilde{I}_{3}-\tilde{I}_{6}-\mathrm{i}\left(\tilde{I}_{18}+\tilde{I}_{22}\right) & 2 \tilde{I}_{13}+2 \mathrm{i} \tilde{I}_{16} \\ \text { (Hermitian conjugate }) & & & I_{1}-I_{5}+I_{11}-I_{9} & 2 I_{8}-2 \mathrm{i} I_{17} \\ & & & & 2 I_{1}-2 \bar{I}_{24}\end{array}\right]$

2.4. The laboratory observables $\left(\theta_{\mathbf{R}}\right.$ is the recoil angle).

$$
\begin{aligned}
& \sigma \quad=I(00 ; \mathrm{oo})=I_{1}, \\
& P \quad=I(n \circ ; \circ o)=I(\mathrm{o} n ; \mathrm{oo})=I(\mathrm{oo} ; \mathrm{o} n)=I(n n ; \mathrm{o} n)=-I_{2} \text {, } \\
& D_{N N}=I(\mathrm{on} ; \mathrm{on})=I_{5} \text {, } \\
& I(s l ; 00)=-I_{8} \text {, } \\
& C_{N N}=I(n n ; 00)=-I_{9}, \\
& I(s s ; \mathrm{oo})=I_{10} \text {, } \\
& K_{N N}=I(\text { no; on })=-I_{11} \text {, } \\
& I(s s ; \text { on })=-I_{17} \text {, } \\
& I(s l ; o n)=I_{23} \text {, } \\
& R \quad=I(\mathrm{o} s ; \mathrm{os})=\tilde{I}_{4} \sin \theta_{\mathrm{R}}-\tilde{I}_{6} \cos \theta_{\mathrm{R}}, \\
& A=I(\mathrm{o} l ; \mathrm{os})=\tilde{I}_{3} \sin \theta_{\mathrm{R}}+\tilde{I}_{4} \cos \theta_{\mathrm{R}} \text {, } \\
& I(n s ; o s)=\tilde{I}_{15} \cos \theta_{\mathrm{R}}-\tilde{I}_{18} \sin \theta_{\mathrm{R}} \text {. } \\
& I(n l ; o s)=\tilde{I}_{15} \sin \theta_{\mathrm{R}}+\tilde{I}_{22} \cos \theta_{\mathrm{R}} \text {, } \\
& I(s \mathrm{O} ; \mathrm{os})=\tilde{I}_{12} \cos \theta_{\mathrm{R}}-\tilde{I}_{13} \sin \theta_{\mathrm{R}} \text {, } \\
& I(s n ; \mathrm{os})=\tilde{I}_{16} \cos \theta_{\mathrm{R}}+\tilde{I}_{19} \sin \theta_{\mathrm{R}} \text {. }
\end{aligned}
$$

Here $I(a b ; c d)$ denotes a laboratory observable, for which the spin components of the beam, target, scattered and recoil particles are determined along the $a, b, c$ and $d$ direction respectively. $a, b, c$ and $d$ may each be any of the directions $s, n$ or $l$ (see fig. 1). A "o" denotes not determined.

\subsection{Numerical estimates for the laboratory observables.}

We obtained numerical estimates for the observables of the previous section from the helicity amplitudes given in ref. 16. We took $P_{\text {lab }}=6 \mathrm{GeV} / c$ and $-t=0.2(\mathrm{GeV} / c)^{2}$. The experimental values for six observables have been listed in brackets.

$$
\begin{aligned}
& I(\mathrm{Oo} ; \mathrm{Oo})=1 \text {, } \\
& \text { [normalized to 1], } \\
& I(\text { no } ; \text { oo })=+0.12 \pm 0.01 \text {, } \\
& {\left[P=0.131 \pm 0.007 \text { at }|t|=0.225\left(\mathrm{GeV} / c^{2}\right]^{6}\right) \text {, }} \\
& I(\mathrm{on} ; \mathrm{on})=0.9996 \pm 0.08 \text {, } \\
& \left.\left[D_{N N}=0.9 \pm 0.11 \text { at }|t|=0.29(\mathrm{GeV} / c)^{2}\right]^{14}\right), \\
& I(s l ; 00)=-0.0015 \pm 0.04, \\
& I(n n ; \mathrm{oo})=+0.0645 \pm 0.04 \text {, } \\
& \left.\left[C_{N N}=\left\{\begin{array}{l}
0.04 \pm 0.02 \\
0.11 \pm 0.02
\end{array}\right\} \text { at }|t|=\left\{\begin{array}{l}
0.17 \\
0.27
\end{array}\right\}(\mathrm{GeV} / c)^{2}\right]^{18}\right) \\
& I(s s ; \infty)=-0.0022 \pm 0.04, \\
& I(n \circ ; \text { on })=+0.0645 \pm 0.08 \text {, } \\
& \left.\left[K_{N N}=0.14 \pm 0.08 \text { at }|t|=0.53(\mathrm{GeV} / c)^{2}\right]^{15}\right), \\
& I(s s ; \text { on })=-0.0012 \pm 0.08 \text {, }
\end{aligned}
$$


$I(s l ; o n)=0.0261 \pm 0.08$,

$I(\mathrm{os} ; \mathrm{os})=-0.198 \pm 0.08$,

$\left.\left[R=-0.17 \pm 0.24 \text { at }|t|=0.23(\mathrm{GeV} / c)^{2}\right]^{20}\right)$

$I(\mathrm{ol} ; \mathrm{o} s)=0.938 \pm 0.08$,

$I(n s ; o s)=+0.03 \pm 0.08$,

$I(n l ; o s)=0.115 \pm 0.08$,

$I(s o ; o s)=0.001 \pm 0.08$,

$I(s n ; o s)=0.0247 \pm 0.08$.

Field and Stevens ${ }^{22}$ ) have compared predictions of various models. They usually agree with the values quoted above, except for some disagreement on the observables which depend on the phases between natural and unnatural parity exchange amplitudes, namely $I(s s ; o o), I(s l, o n), I(s o ; o s)$ and $I(s \mathrm{~s} ; o s)$. 